\title{
Estudio de evaluación agronómica en el cultivo de papa (Solanum spp.) con tolerancia al déficit hídrico -
} Ecuador

\section{Study of agronomic evaluation in the culture of papa (Solanum spp.) with tolerance to the water deficit - Ecuador}

\author{
Fabricio Peña Robinson ${ }^{1 *} \mathbb{D}$, Juan Eduardo León Ruiz'미, Vicente Javier Parra León
}

\section{RESUMEN}

El presente estudio da a conocer el comportamiento agronómico de seis genotipos (INIAP Estela, INIAP Natividad, Superchola, clon 10-10-97, clon $07-29-11$ y clon $98-02-06)$, mediante el factor de riego a través de dos métodos: 1) condiciones óptimas durante todo el ciclo de cultivo (con riego); 2) suspensión del riego en la etapa fenológica de floración por un periodo de 20 días (sin riego). Esto permite la cuantificación del requerimiento hídrico, y determinar el genotipo con mejor tolerancia al déficit híbrido de la evaluación agronómicamente de seis genotipos de papa (Solanum spp). Se evaluaron los siguientes parámetros: contenido gravimétrico de agua en el suelo, potencial mátrico del suelo, evapotranspiración del cultivo de referencia (Eto), evapotranspiración del cultivo (Etc), datos que permitieron determinar los requerimientos hídricos por estado fenológico (Kc). Las variables para identificar el grado de tolerancia al déficit hídrico fueron: contenido de clorofila, contenido relativo de agua, número de tubérculos por plantas, rendimiento por planta, rendimiento por tamaño de tubérculo, rendimiento total y materia seca del tubérculo. Se concluye que las variedades INIAP- Estela, INIAP Natividad y los clones 98-02-06, 10-10-97 presentaron valores altos por los que las considera tolerantes a la sequía.

Palabras clave: tolerancia al déficit híbrido, cuantificación, requerimientos hídricos, Ecuador.

\begin{abstract}
The present study shows the agronomic behavior of six genotypes (INIAP Estela, INIAP Natividad, Superchola, clone 10 - 10 - 97, clone $07-29$ - 11, and clone 98 - 02 - 06), using the irrigation factor through two methods: 1) optimal conditions during all the crop cycle (with irrigation);2) suspension of irrigation in the phenological stage of flowering for 20 days (without irrigation). This allows quantifying the water requirement and determining the genotype with the best tolerance to the hybrid deficit from the agronomic evaluation of six potato genotypes (Solanum spp). The following parameters were evaluated: soil gravimetric water content, soil matric potential, evapotranspiration of the reference crop (Eto), evapotranspiration of the crop (Etc), data that allowed determining water requirements by phenological state $(\mathrm{Kc})$. The variables to identify the degree of tolerance to the hydric deficit were: chlorophyll content, relative water content, number of tubers per plant, yield per plant, yield per size of tuber, total yield, and dry matter tuber. It is concluded that the varieties INIAP-Estela, INIAP Natividad, and the clones 9802-06, 10-10-97 presented high values for which it considers them tolerant to drought.
\end{abstract}

Keywords: hybrid deficit tolerance, quantification, water requirements, Ecuador. 


\section{INTRODUCCIÓN}

Tanto Ecuador, como varios países del mundo están siendo afectados por el cambio climático. Amenazando principalmente al rendimiento de los cultivos agrícolas, ocasionado un riesgo para la seguridad alimentaria de los países (Nina et al 2016). Se habla, que la temperatura alrededor del mundo podría incrementarse gradualmente hasta $6^{\circ} \mathrm{C}$ en el 2050. Donde se evidenciará un fuerte cambio en los "pequeños agricultores" o de "agricultura de subsistencia" de países en desarrollo como es el caso de Ecuador (Morton, 2007). Mediante estos acontecimientos que están pasando, surgen nuevas investigaciones. Por lo que, el desarrollo de genotipos adaptados a condiciones hídricas cambiantes, es esencial para contribuir a la seguridad alimentaria de una población creciente, que requiere incrementos significativos en la producción de alimentos (Polanía et al., 2009). La producción de los cultivos bajo el efecto del cambio climático, principalmente en el escenario de sequía, presentarán reducciones en rendimiento, incremento en el costo de producción y consecuentemente hambre y desplazamiento (Herrera et al., 2012).

Entre estos tenemos al cultivo de papa (Solanum spp.) que es un alimento diario en la dieta de las personas que viven en la provincia de Chimborazo, donde la principal economía depende de la agricultura. La planta para que se desarrolle depende de las condiciones climáticas, suelo y otros factores (Altieri y Nicholls, 2013). La cantidad de agua requerida para la papa varía de 400 a $800 \mathrm{~mm}$ por cultivo, y al presentar etapas críticas de necesidad de agua, siendo estos periodos de emergencia los que retrasan la tuberización, evidenciándose un efecto negativo en el rendimiento provocado por un estrés hídrico más acentuado en las plantas, ocasionando una disminución en el volumen de los tubérculos y, por lo tanto, una disminución en el tamaño de los mismos (Gabriel et al 2013). Siendo el principal afectado por este cambio el agricultor.

\section{MATERIAL Y MÉTODOS}

\section{Materiales}

La investigación se realizó en el Campus Macají, de la Escuela Superior Politécnica de Chimborazo (ESPOCH), Cantón Riobamba, Provincia de Chimborazo. En la latitud $1^{\circ} 39^{\prime} 18,82^{\prime \prime} \mathrm{S}$ y la longitud 7840'39.99"O; a una altitud de 2.821 m.s.n.m.

Las condiciones climáticas de la zona son una temperatura media anual de $13,5^{\circ} \mathrm{C}$, una precipitación media anual de $350 \mathrm{~mm} /$ año y una humedad relativa del 59,6 \%. Según el MAE (2012) clasificación ecológica es de Bosque Siempre Verde Montano del norte y centro de la cordillera oriental de los Andes.

\section{Metodología}

Se realizaron dos manejos, el primero en condiciones favorables para el desarrollo de cultivo "Con Riego" y el segundo con un aporte hídrico reducido en el estado fenológico de floración "Sin Riego". Instalándose en dichos manejos un sistema de riego por goteo para evaluar la capacidad de recuperación de las plantas después del déficit hídrico.

Se utilizó el diseño de parcela dividida, en donde la parcela grande fue el factor riego (Con riego y Sin riego) y las parcelas pequeñas fueron los genotipos de la siguiente manera: Número de unidades experimentales 36, Numero de tratamientos 12, Numero de repeticiones 3 . El área total del experimento fue de $492 \mathrm{~m} 2$, con un largo del ensayo de $24 \mathrm{~m}$, un ancho del ensayo de $18 \mathrm{~m}$ y una Distancia entre repeticiones de $0,5 \mathrm{~m}$, contando con 5 caminos.

Las características de la parcela experimental fueron las siguientes:

- Número de tubérculos / parcela $=40$

- Número de $\operatorname{surcos}=4$

- $\quad$ Ancho de la parcela $=3 \mathrm{~m}$

- $\quad$ Largo de la parcela $=4 \mathrm{~m}$

- Distancia entre surcos $=1 \mathrm{~m}$

- Distancia entre plantas $=0,3 \mathrm{~m}$

- Número de plantas evaluadas $=16$

- Área neta de parcela $=4,8 \mathrm{~m} 2$

- Área total de la parcela $=12 \mathrm{~m} 2$

- $\quad$ Forma de parcela = rectangular. 
Las semillas de seis genotipos (clones y variedades) de papa (Solanum spp.) del PNRT - INIAP fueron:

- G1 Estela (Variedad testigo tolerante)

- G2 Natividad (Variedad testigo)

- G3 Superchola (Variedad testigo susceptible)

- $\mathrm{G} 4 * 10-10-97$ (Bolona x (CHS625 x S. pausissectum)
- $\mathrm{G} 5 * 07-29-11$ (ASO861 x HSO213)

- $\mathrm{G6} * 98-02-06$ (INIAP Gabriela x INIAP Margarita).

Para la obtención de datos de campo se realizó con un esquema de análisis estadístico de varianza para un diseño de parcela dividida con una interrelación entre el factor riego y los genotipos de papa en estudio (Tabla 1).

Tabla 1. Esquema de análisis de varianza para la evaluación de genotipos de papa (Solanum spp.)

\begin{tabular}{ccc}
\hline Fuentes de variación & Formulas & Grados de libertad \\
\hline Repeticiones & $r-1$ & 2 \\
A (Riego) & $a-1$ & 1 \\
Error A & $(a-1) \times(r-1)$ & 2 \\
B (Genotipos) & $b-1$ & 5 \\
Interacción AxB & $(a-1) \times(b-1)$ & 5 \\
Error B & $a(b-1) \times(r-1)$ & 20 \\
Total & $(a \times b \times r)-1$ & 35 \\
\hline
\end{tabular}

Tras el análisis de varianza, se realizó un análisis funcional para los factores e interacciones. Para ello se utilizó la prueba de significación de Tukey al 5\%.

\section{RESULTADOS}

A continuación se presentan las condiciones climáticas dadas en el proceso de siembra del cultivo de papa, durante los meses de mayo y septiembre:

Durante los meses de mayo a septiembre del 2018, la temperatura máxima fue de $21^{\circ} \mathrm{C}$ en mayo hasta $26,4^{\circ} \mathrm{C}$ en septiembre. En cambio, la temperatura mínima fue en mayo de $12,1^{\circ} \mathrm{C}$ hasta $5,9^{\circ} \mathrm{C}$ en septiembre. Respecto la precipitación, en mayo se registró un valor de $0,85 \mathrm{~mm}$, bajó a 0,3 mm en junio, luego $0,5 \mathrm{~mm}$ en julio, $0,11 \mathrm{~mm}$ en agosto y finalmente descendió a $0 \mathrm{~mm}$ en septiembre. Por otro lado, los valores mensuales promedios del viento se encontraron entre 0,31 a $1,18 \mathrm{~m} / \mathrm{s}$, considerándola como "no perjudicial" para el desarrollo de los genotipos de papa (Solanum spp.). En el caso de la humedad relativa, el promedio fue reduciéndose de $69,6 \%$ en mayo a 63,6 $\%$ en septiembre, con un ligero incremento en agosto $(67,26 \%)$, que no representó ningún efecto negativo para el desarrollo de plagas y enfermedades.

Requerimiento hídrico total (mm/ciclo) de los seis genotipos de papa (Solanum spp)

La variedad INIAP - Estela, en el manejo "Con Rie- go", la duración del ciclo fue de 139 días, con un requerimiento hídrico de $416,7 \mathrm{~mm} / \mathrm{m}^{2} /$ ciclo fenológico y una lámina de riego promedio de $19 \mathrm{~mm}$ (19 litros $/ \mathrm{m}^{2}$ ). Con el manejo “Sin Riego", la duración del ciclo fue de 138 días, con un requerimiento hídrico de $331 \mathrm{~mm} / \mathrm{m}^{2} /$ ciclo fenológico y una lámina de riego promedio de $15 \mathrm{~mm}$ (15 litros $\left./ \mathrm{m}^{2}\right)$.

La variedad INIAP - Natividad, en el manejo "Con Riego", la duración del ciclo fue de 141 días, con un requerimiento hídrico de $410,4 \mathrm{~mm} / \mathrm{m}^{2} /$ ciclo fenológico y una lámina de riego promedio de $17,75 \mathrm{~mm}$ $\left(17,75\right.$ litros $\left./ \mathrm{m}^{2}\right)$. Con el manejo "Sin Riego", la duración del ciclo fue de 138 días, requerimiento hídrico de $323 \mathrm{~mm} / \mathrm{m}^{2} /$ ciclo fenológico y una lámina de riego promedio de 15,25 $\mathrm{mm}\left(15,25\right.$ litros $\left./ \mathrm{m}^{2}\right)$.

La variedad Superchola, en el manejo "Con Riego", la duración del ciclo fue de 144 días, con un requerimiento hídrico de $410 \mathrm{~mm} / \mathrm{m}^{2} /$ ciclo fenológico y una lámina de riego promedio de $20 \mathrm{~mm}$ (20 litros $\left./ \mathrm{m}^{2}\right)$. Con el manejo "Sin Riego", la duración del ciclo fue de 140 días, con un requerimiento hídrico de $324 \mathrm{~mm} / \mathrm{m}^{2} /$ ciclo fenológico y una lámina de riego promedio de 15,25 $\mathrm{mm}\left(15,25\right.$ litros $\left./ \mathrm{m}^{2}\right)$.

El clon 07 - 29 - 11, en el manejo "Con Riego", la duración del ciclo fue de 140 días, con un requerimiento hídrico de $409 \mathrm{~mm} / \mathrm{m}^{2} /$ ciclo fenológico y una lámina de riego promedio de 22,25 $\mathrm{mm}\left(22,25\right.$ litros $\left./ \mathrm{m}^{2}\right)$. 
Con el manejo "Sin Riego", la duración del ciclo fue de 138 días, con un requerimiento hídrico de 331 $\mathrm{mm} / \mathrm{m}^{2} /$ ciclo fenológico y una lámina de riego promedio de $17,25 \mathrm{~mm}\left(17,25\right.$ litros $\left./ \mathrm{m}^{2}\right)$.

El clon 98 - 02 - 06, en el manejo "Con Riego", la duración del ciclo fue de 139 días, con un requerimiento hídrico de $410 \mathrm{~mm} / \mathrm{m}^{2} /$ ciclo fenológico y una lámina de riego promedio de 21,25 mm (21,25 litros $\left./ \mathrm{m}^{2}\right)$. Con el manejo "Sin Riego", la duración del ciclo fue de 138 días, con un requerimiento hídrico de 331 $\mathrm{mm} / \mathrm{m}^{2} /$ ciclo fenológico y una lámina de riego promedio de $15,5 \mathrm{~mm}\left(15,5\right.$ litros $\left./ \mathrm{m}^{2}\right)$.

El clon 10 - 10 - 97, en el manejo "Con Riego", la duración del ciclo fue de 139 días, con un requerimiento hídrico de $409 \mathrm{~mm} / \mathrm{m}^{2} /$ ciclo fenológico y una lámina de riego promedio de 21,75 mm (21,75 litros $\left./ \mathrm{m}^{2}\right)$. Con el manejo "Sin Riego", la duración del ciclo fue de 136 días, con un requerimiento hídrico de 332 $\mathrm{mm} / \mathrm{m}^{2} /$ ciclo fenológico y una lámina de riego promedio de $16,5 \mathrm{~mm}\left(16,5\right.$ litros $\left./ \mathrm{m}^{2}\right)$.

Por lo tanto, tomando en consideración lo antes mencionado, el genotipo con mayor requerimiento hídrico durante todo el ciclo de cultivo fue INIAP - Estela con $416,7 \mathrm{~mm} /$ ciclo, valor que se encuentra debajo del rango indicado por Haapala (2008), que es de 500 a $700 \mathrm{~mm}$, es decir, un requerimiento hídrico menor, lo que indica un grado positivo para ser implementado en zonas agrícolas con precipitaciones entre $400-450 \mathrm{~mm}$.

\section{Humedad del suelo}

En el manejo "Con Riego" se observó que la disponibilidad de humedad a diferentes profundidades $(15,30$ y $45 \mathrm{~cm}$ ) se mantuvo entre humedad disponible y capacidad de campo, permitiendo que a medida que el sistema radicular de los genotipos crecía toda el agua de la capa del suelo sea disponible, para compensar las pérdidas debido a la evapotranspiración (Figura 1).

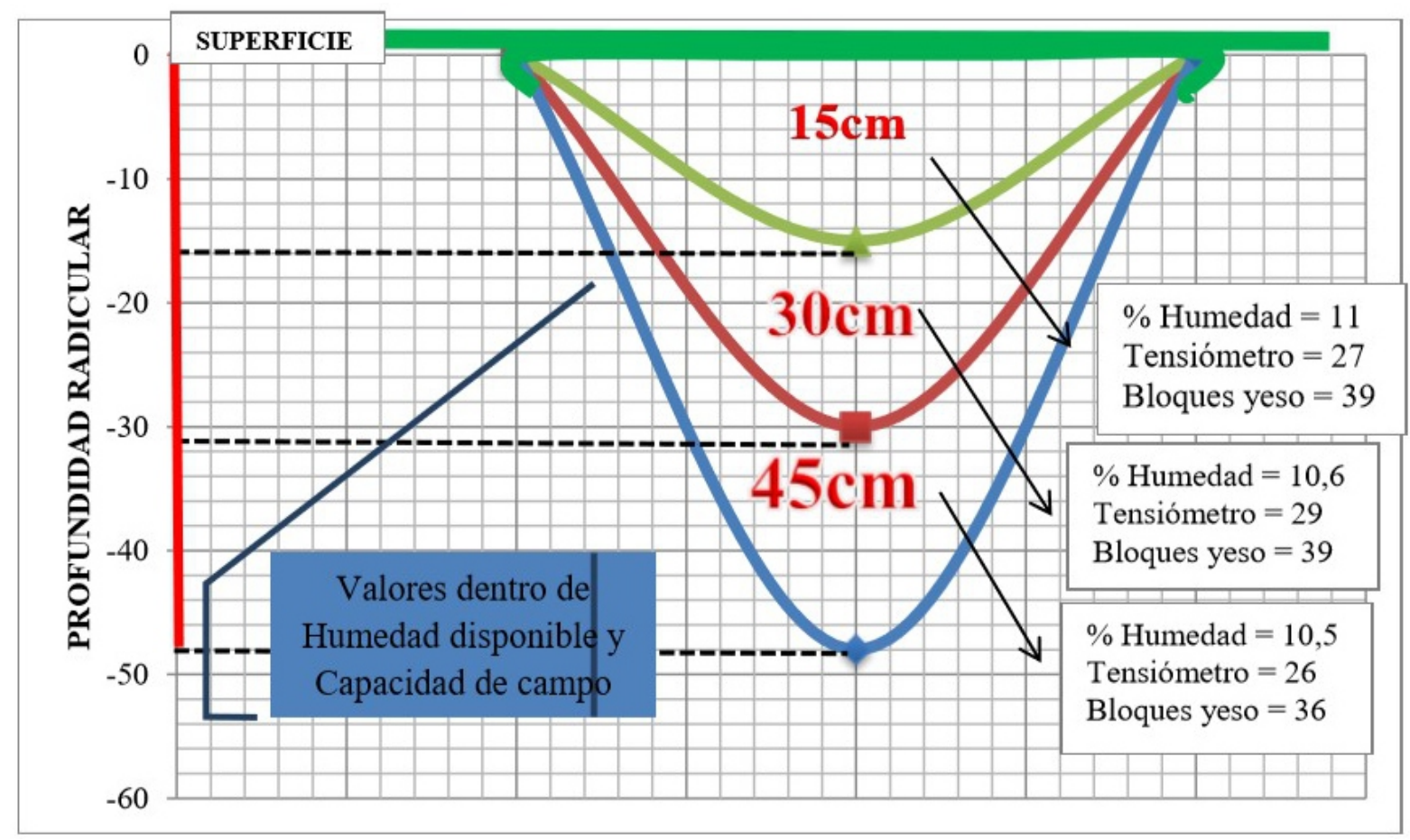

Figura 1. Humedad del suelo y profundidad radicular del manejo "Con Riego"

En el manejo "Sin Riego", la disponibilidad de humedad aprovechable en las diferentes profundidades fue variable y bajo del rango óptimo, por lo que se debe controlar regularmente el nivel de humedad del suelo para determinar cuándo regar y qué cantidad de agua se debe aplicar, la papa es vulnerable ante el déficit hídrico durante su ciclo de crecimiento, dando como consecuencia bajo rendimiento y mala calidad del tubérculo, poniendo en manifiesto el importante papel que cumple este elemento, enfatiza (Figura 2). 


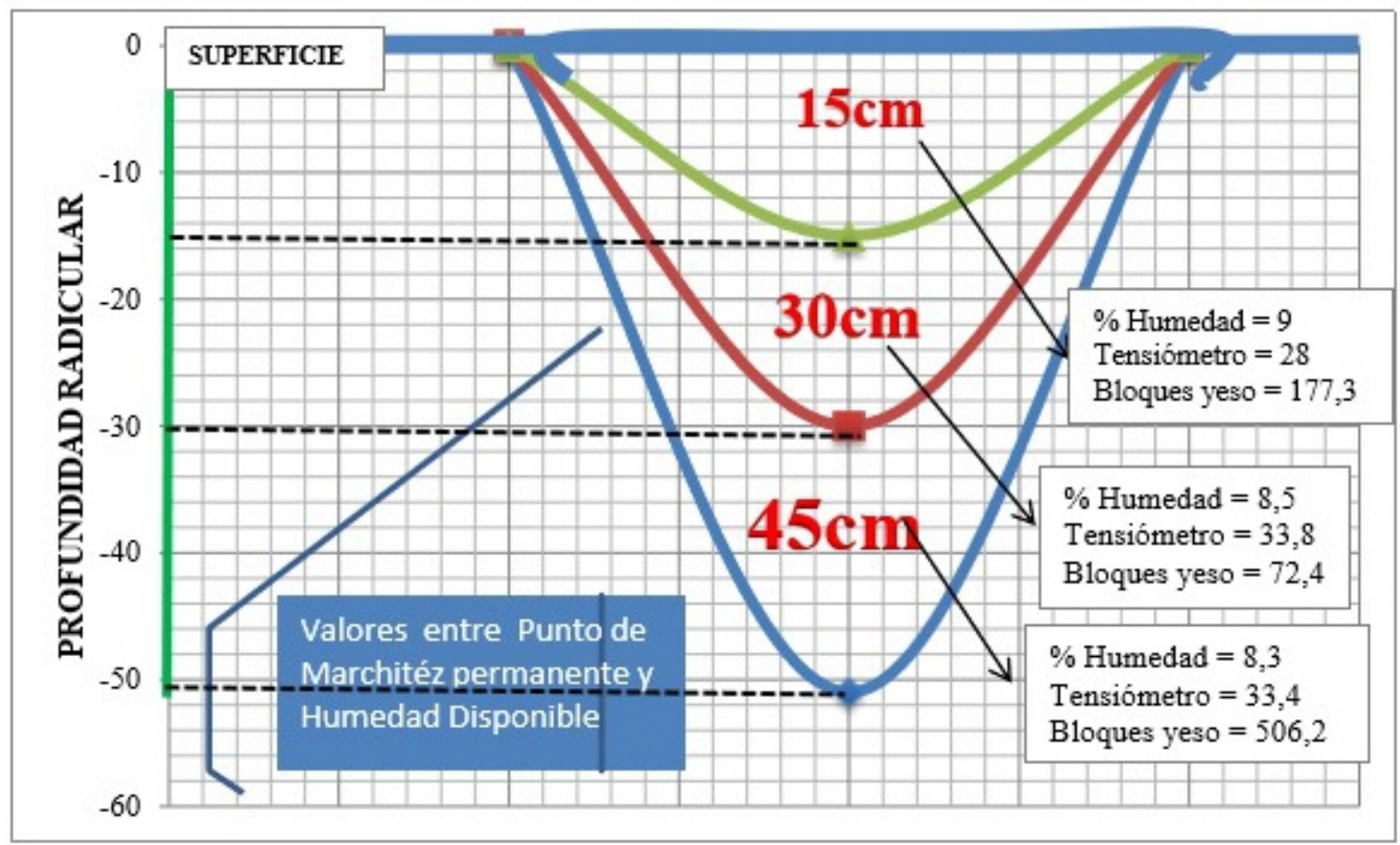

Figura2. Humedad del suelo y profundidad radicular del manejo "Sin Riego"

\section{Emergencia}

Según el análisis de varianza, para el porcentaje de emergencia de los genotipos con tolerancia al déficit hídrico a los 45 días después de la siembra, no expresa diferencias significativas para ninguna de las fuentes de variación, de-bido a que la dotación de agua fue similar para todos los tratamientos y se utilizó semilla de buena calidad. El promedio general de $95,56 \%$ y el coeficiente de variación 4,45\%.

Las diferencias numéricas observadas entre los genotipos, se deben exclusivamente a las características de la semilla de cada uno de los genotipos variando entre 91,7 al $100 \%$ (Figura 1S).

\section{Altura de planta}

Altura de planta a los 45 días: Según el análisis de varianza de los genotipos evaluados para la altura de planta a los 45 días después de la siembra da como resultado un promedio general de $29,9 \mathrm{~cm}$ y el coeficiente de variación $16,94 \%$.

Las diferencias de altura observadas, en el no fueron influenciadas por el riego ya que a los dos métodos hasta ese momento se los manejó por igual (Figura 2S). Altura de planta a los 75 días: Según el análisis de varianza de los genotipos evaluados para la altura de planta a los 75 días después de la siembra, da como resultado un promedio general de $68,55 \mathrm{~cm}$ y el coeficiente de variación $4,6 \%$

En esta etapa los genotipos empiezan a sentir el déficit hídrico, observando que la variedad Superchola en el manejo “Con Riego" presenta una altura de 74,9 cm, a diferencia de los genotipos 07 - 29- 11 con una altura de 65,03 cm. Manejo "Sin Riego", 98-02-06 con una altura de $64,83 \mathrm{~cm}, 07-29-11$ con una altura de 64,33 cm y $10-10-97$ con una altura de $62,70 \mathrm{~cm}$, mientras que el resto de los genotipos se encontraron con valores intermedios (Figura $3 \mathrm{~S}$ ).

Altura de planta a los 105 días: Según el análisis de varianza de los genotipos evaluados para la altura de planta a los 105 días después de la siembra, da como resultado un promedio general de $73,33 \mathrm{~cm}$ y el coeficiente de variación 5,55\%.

Se pudo apreciar, que la variedad Superchola en el manejo "Con Riego" presenta la mayor altura con 84,13 cm. Manejo “Sin Riego" en cambio el clon 10 10 - 97 presentó el menor crecimiento, con una altura de 64,97 cm consecuencia de lo cual se lo ubicó en el 
ultimo (Figura 4S).

\section{Cobertura del suelo}

En el manejo "Con Riego" y "Sin Riego", se obtuvo que las variedades INIAP - Estela, INIAP - Natividad, Superchola y el clon $98-02-06$ presentan la mayor cobertura del suelo con valores de 3 y los tratamientos: clon 07 - 29 - 11 y 10 - 10 - 97 una cobertura buena con valor de 2 , lo cual permite manifestar que el estrés hídrico no afecta la cobertura de ninguno de los seis genotipos.

\section{Vigor de la planta}

En el manejo "Con Riego" y "Sin Riego" las variedades INIAP-Estela, INIAP-Natividad, Superchola y el clon $98-02-06$ presentaron el mayor vigor con una calificación de "vigorosa", es decir los genotipos mostraron frondosidad y cubrieron el surco. Por otra parte, el clon $07-29$ - 11, tuvo una calificación de "medio" es decir presentó media frondosidad y cubrió la mitad del surco. Por último, el clon 10 - 10 - 97, quien fue reducido de un grado 3 (vigorosa) en "Con Riego" a un grado 2 (medio) “Sin Riego".

\section{Floración}

Según el análisis de varianza para días a la floración da como resultado: promedio general de 70 días y el coeficiente de variación $8,9^{-8} \%$.

Los tratamientos "Con Riego" se demoran entre 1 a 2 días más que los tratamientos "Sin Riego" en alcanzar la floración (Figura 5S).

\section{Inicio de tuberización}

Según el análisis de varianza da como resultado: un promedio general fue de 85 días y el coeficiente de variación $1^{-7} \%$.

Se observó que en el manejo "Con Riego" el clon 07 29-11 llego a tuberización a los 97 días y se ubicó en el mayor valor. Mientras que en el manejo "Sin Riego" la variedad INIAP - Natividad, inició la tuberización a los 70 días y se ubicó en el menor valor (Figura 6S).

\section{Senescencia}

Según el análisis de varianza para días a la senescencia da como resultado: un promedio general de 139 días y el coeficiente de variación $1,07 \%$.

En el manejo "Con Riego" el genotipo más tardío fue la variedad Superchola y en el manejo "Sin Riego" el genotipo más precoz fue el clon $10-10$ - 97 con 136 días, los demás genotipos presentaron valores intermedios (Figura 7S).

\section{Potencial de recuperación}

En el manejo "Con Riego" las condiciones eran óptimas para que los genotipos siempre se mantuvieron turgentes con un valor en la escala de 8 a 9 ( 80 a mayor $95 \%$ de turgencia). Todo lo contrario, se dio en el manejo "Sin Riego", notándose diferencias en el proceso de transpiración entre los genotipos, ya que algunos perdieron agua más rápidamente que otras.

Destacando en "déficit hídrico" la variedad INIAP Estela, que presentó mayor tolerancia soportando un bajo potencial hídrico, manteniendo la turgencia y activo los procesos de crecimiento, desarrollo y producción debido a la acumulación activa de solutos, llegando hasta valores de $6(60 \%)$. Los efectos más notorios se observaron en los clones $98-02-6$ y $10-$ 10 - 97 llegando en la escala hasta valores de 4 (40\%), pero posterior a la "recuperación" de agua no alcanzaron sus valores iniciales de turgencia (9 o mayor 95 \%), afirmando lo expuesto Ackerson (1997), quien señala que la gran sensibilidad de las plantas a déficit hídricos, se debe a la poca capacidad de recuperación, luego de finalizado el período de estrés (Figura 8S).

\section{Contenido de clorofila}

Según el análisis de varianza para el contenido de clorofila, antes del déficit se tuvo como resultado un promedio general de 46,04 SPAD, con un coeficiente de variación de 3,88\%. En "déficit" da como resultado un promedio general de 48,43 SPAD, y un coeficiente de variación 4,93\%. En "recuperación" da como resultado un promedio de 47,13 SPAD, y el coeficiente de variación $3,35 \%$.

En cuanto al contenido de clorofila "antes del déficit", destaca el clon 98-02-06 "Sin Riego", que presentó el mayor contenido con 49,73 SPAD. En cambio, la variedad INIAP - Estela "Con Riego" presentó el menor contenido con 42,33 SPAD.

En "déficit hídrico", el clon 98 - 02 - 06 "Sin Riego" fue el que destacó con mayor contenido con 54,03 
SPAD. Todo contrario se dio en la variedad INIAP Estela “Con Riego", que presentó el menor contenido con 44,17 SPAD.

En "recuperación” el mayor contenido de clorofila, lo mostró el clon 98 - 02 - 06 "Sin Riego" con 50,70 SPAD. Seguido del mismo genotipo "Con Riego" con 49,37 SPAD y del clon 10 - 10 - 97 “Sin Riego" con 48,87 SPAD. Por otro lado, el menor contenido de clorofila fue la variedad INIAP - Estela "Con Riego" con 41,27 SPAD.

También queda de manifiesto que el mayor contenido de clorofila en las tres etapas de evaluación lo obtuvo el clon 98-02-06 "Sin Riego" y el menor la variedad INIAP-Estela “Con Riego"(Figura 9S).

\section{Contenido relativo de agua en hojas(wrc)}

Según el análisis de varianza para el contenido relativo de agua en las hojas "antes de déficit hídrico", dio como resultado un promedio general de $76,49 \%$, y un coeficiente de variación del 7,67\%. En "déficit hídrico", el promedio general fue de $74,43 \%$, con un coeficiente de variación del 6,35\%. En "recuperación" el promedio general fue de $77,7 \%$, con un coeficiente de variación del $6,96 \%$.

El contenido relativo de agua "antes del déficit hídrico", destacó la variedad Superchola "Con Riego" al presenta el mayor contenido con un 83,83\%. En cambio, el clon 07 - 29 - 11 "Sin Riego" presento el menor contenido con un $65,57 \%$.

En "déficit hídrico" volvió a destacar la variedad Superchola "Con Riego", que presentó el mayor contenido con un $85 \%$. Mientras que el clon $07-29-11$ "Sin Riego", tuvo el menor contenido con un $62,17 \%$. Después de estar sometidos los genotipos al déficit hídrico en el manejo "Sin Riego" se les dotó de láminas grandes de agua para lograr la "recuperación", destacando el caso de la variedad INIAP - Estela (Figura 10S).

\section{Materia seca de la planta completa}

Según el análisis de varianza para el porcentaje de materia seca de la planta completa dio como resultado un promedio general de $21,03 \%$ y un coeficiente de variación $27,17 \%$.
La variedad INIAP - Estela "Con Riego" tuvo el mayor contenido de materia seca con un $26 \%$. Mientras que el menor contenido de materia seca, fue el clon 07-29-11 “Sin Riego" con un 16\% (Figura 11S).

\section{Rendimiento y sus componentes}

Tubérculos por planta: según el análisis de varianza para el número de tubérculos por planta dio como resultado un promedio general de 26,36 unidades y un coeficiente de variación del 16,11\%.

En el manejo “Con Riego” destacó el clon 07-29-11, que tuvo el menor número de tubérculos con 17 unidades. Mientras que en el manejo "Sin Riego", fue la variedad Superchola la que presentó el mayor número de tubérculos por planta con un número de 38 unidades (Figura 12S).

Rendimiento por planta: Según el análisis de varianza para el rendimiento por planta dio como resultado un promedio general de $0,8 \mathrm{~kg} /$ planta y un coeficiente de variación del 23,07\%.

El genotipo con manejo "Con Riego", que presentó mayor rendimiento por planta $(\mathrm{kg} / \mathrm{planta})$, fue INIAP Estela con una media de 1,01 Kg. Por el contrario, el genotipo con manejo "Sin Riego" , que presento menor rendimiento fue el clon $07-29-11$, con 0,42 Kg. Destacar también que el clon 98 - $02-06$ se mantuvo dentro de un mismo rango en los dos tipos de manejo (Figura 13S).

Rendimiento por tamaño del tubérculo: según el análisis de varianza para el rendimiento de papa en categoría "comercial" dio como resultado un promedio general de $1,83 \mathrm{~kg}$ y un coeficiente de variación del $48,97 \%$. Para el rendimiento de papa categoría “semilla" dio como resultado un promedio general de 6,94 $\mathrm{kg}$ y un coeficiente de variación del 19,62\%. Para rendimiento de papa categoría "no comercial" dio como resultado un promedio general de $3,04 \mathrm{~kg}$ y un coeficiente de variación del 27,52\%.

Para el rendimiento de papa en categoría "comercial", el clon 10 - $10-97$ “Con Riego” presentó el mayor rendimiento con 4,43 $\mathrm{kg}$. En cambio, la variedad Superchola "Sin Riego" no presentó producción.

En el rendimiento de papa categoría "semilla", la 
variedad Superchola “Con Riego" presentó el mayor rendimiento con 10,47 kg. Por el contrario, el clon $07-$ 29 - 11 "Sin Riego" presentó el menor rendimiento con $4,07 \mathrm{~kg}$.

Para el rendimiento de papa categoría "no comercial", los genotipos Superchola, INIAP - Natividad, INIAP - Estela y el clon 10 - 10 - 97 “Sin Riego" presentaron el mayor rendimiento con 4,73 kg, 4,67 kg, 3,97 kg y $3,93 \mathrm{~kg}$ respectivamente. En cambio, en el manejo "Con Riego" dichos genotipos obtuvieron menores rendimientos, con $2,87 \mathrm{~kg}, 2,80 \mathrm{~kg}, 2,53 \mathrm{~kg}$ y 2,27 respectivamente (Figura 14S).

Rendimiento total: según el análisis de varianza para el rendimiento total da como resultado un promedio general de $11,67 \mathrm{~kg}$ y el coeficiente de variación 16,93 $\%$.

Los genotipos Superchola, INIAP - Natividad, INIAP - Estela y los clones 98 - 02 - 06, 10 - 10 - 97 “Con Riego" presentaron el mayor rendimiento con 14,53 $\mathrm{kg}, 13,90 \mathrm{~kg}, 13,73 \mathrm{~kg}, 13,67 \mathrm{~kg}$ y $13,47 \mathrm{~kg}$ respectivamente, en cambio el clon $07-29$ - 11 "Sin Riego" presentó el menor rendimiento con 6,4 kg (Figura $15 \mathrm{~S})$.

Porcentaje de materia seca del tubérculo: Según el análisis de varianza para el porcentaje de materia seca del tubérculo da como resultado un promedio general de 23, $89 \%$ y el coeficiente de variación 8,01\%.

Señala que la variedad de superchola "Con Riego" tubo un valor de $25 \%$ de materia seca y el clon $07-29$ - 11 “Sin Riego" presentó el mayor contenido de materia seca con $26,70 \%$, a diferencia de la variedad INIAP - Natividad "Sin Riego" presentó el menor contenido de materia seca con 20,87\% (Figura 16S).

\section{Selección de variables}

La selección de variables que permitieron identificar un mejor comportamiento de los genotipos de papa (Solanum spp.) con mayor tolerancia al déficit hídrico fueron el contenido de clorofila, contenido relativo de agua en las hojas, materia seca de la planta completa y el rendimiento categoría comercial en el manejo "Sin Riego", ya que en el manejo "Con Riego" fue todo por igual.

\section{Selección de genotipos}

Para la selección de genotipos se destaca los mayores contenidos de clorofila que fueron los clones $98-02-$ 06, $07-29$-11 y las variedades Superchola e INIAP Estela. En el caso del contenido relativo de agua los mejores fueron las variedades INIAP - Natividad, INIAP - Estela y los clones 98 - 02 - 06, 10 - 10-97. La materia seca de la planta completa destaca INIAP Estela, INIAP - Natividad, 10 - 10 - 97 y 98 - 02- 06. El rendimiento en papa comercial los mejores resultados fueron para 98 - 02- 06, INIAP - Estela, 07 - 29 11 y $10-10-97$.

En base a lo señalado, los genotipos seleccionados fueron INIAP - Estela, 98-02 -06, INIAP - Natividad, $10-10-97$, ya que sobre todo en rendimiento por categoría comercial y semilla, presentaron los mejores valores por hectárea. Siendo este un indicador más relevante para tomar en consideración esta selección.

\section{IV.DISCUSIÓN}

En Argentina determino que si existe variabilidad entre diez genotipos utilizados en ensayos de estrés hídrico, donde se realizaron tres tratamientos: control (C): las plantas siguieron un esquema de riego normal, sequía con déficit (SD): a partir del día 45 después de plantación se aplicó riego con déficit (mitad de capacidad de campo), sequía severa (SS): a partir del día 45 después de plantación, no se aplicó riego) con estos parámetros se determinó que las variedades Sipancachi, Unknown y CIS 1802 norte produjeron tubérculos bajo estrés hídrico y la variedad Unknown fue la de mayor peso de tubérculo (Bedogni et al 2009). bi

Referente al como comportamiento agronómico de genotipos de papa (Solanum Spp.) bajo estrés hídrico en invernadero en Ecuador, destaca la investigación realizada en la Estación "Santa Catalina" del Instituto Nacional de Investigaciones Agropecuarias "INIAP". Donde los genotipos utilizados fueron 20 variedades nativas donde el factor estrés hídrico, fue evaluado mediante dos niveles uno con riego durante todo el ciclo e1 (sin estrés) y el otro con una suspensión paulatina del riego al comienzo de la tuberización e2 (con 
estrés). Concluyendo que las variedades Bolona, Leona Negra y Puca Huayro tuvieron un buen comportamiento bajo estrés hídrico en invernadero (Hinojosa et al 2010).

Por otro lado, en Perú se realizó la evaluación de la actividad enzimática usando tres variedades resistentes al estrés hídrico por sequía y cuatro variedades o clones susceptibles. Determinado que las variedades Aurora, Victoria, Jaspe y Salomé pueden ser utilizadas en ambientes donde hay escasez de lluvias, y también como fuente valiosa de genes para transferir a otras variedades (Gabriel, 2013).

Por último, en Chile, se evaluaron tres variedades. La Chona negra, una variedad nativa procedente del banco de germoplasma de la papa de la Universidad Austral de Chile y dos variedades comerciales, Desirée y Karú INIA. Los tratamientos utilizados fueron (control irrigado y un tratamiento sin riego (solo lluvia)), y dos regímenes de temperatura (control y un tratamiento de incremento de temperatura de $3-5^{\circ} \mathrm{C}$ sobre el control) en donde se llegó a la conclusión de que las tres variedades son de buena calidad, y que además soportaron las distintas evaluaciones investigadas (Quintana-Rodríguez, 2018)

Recabando la información se puede identificar que la papa es uno de los cultivos más investigados actualmente, no solo en temas de mejoramiento también en cuidar y preservar la semilla en periodos de tiempo más largo por las condiciones ambientales que se están presentando, por lo que el estudio realizado en Chimborazo sobre el déficit hídrico identifico a 4 variedades de genotipos que son aptos, en otros países como es el caso de Argentina, Perú donde casi existe un promedio de 3 a 4 variedades que soportan el déficit hídrico, ya que uno de los productos más consumidos en América del Sur.

Unos de los detalles que deben ser también tomados en cuenta es la socialización de los datos obtenidos ya que los agricultores, son los que aun después de haberse hechos varios estudios la mayoría de ellos los desconoce y debería ser primordial ya que son ellos los que se beneficiarían directamente con las investigaciones.

\section{V.CONCLUSIÓN}

Los requerimientos hídricos en $\mathrm{mm} /$ ciclo fueron 416,7 para la variedad INIAP - Estela, 410 para la variedad INIAP - Natividad, Superchola y el clon 98-02-06 y 409 para los clones $07-29$ - 11 y $10-10-97$. El rendimiento de tubérculo categoría "comercial "es un indicador relevante para determinar la tolerancia al déficit hídrico al igual que el contenido relativo de agua en las hojas. Bajo condiciones de déficit hídrico el clon 98-02-06 presenta el mayor rendimiento en categoría "comercial” (1,50 kg/planta), seguido de la variedad INIAP-Estela (1.13 kg/planta). Los genotipos con mayor tolerancia al déficit hídrico son INIAP-Estela, 98- 02- 06, INIAP - Natividad y 10 10 - 97. El mejoramiento genético permite incrementar la tolerancia al déficit hídrico lo cual se demuestra en los genotipos estudiados.

\section{REFERENCIAS BIBLIOGRÁFICAS}

Ackerson R.C., D. R. Krieg, T.D. Miller, y R.G Stevens. 1997. "Water relations and physiological activity of potatoes." Journal of The American Society of Horticultural Science $102(5): 572-575$.

Altieri M. A. y C. I. Nicholls. 2013. "Agroecología y resiliencia al cambio climático: principios y consideraciones metodológicas". Agroecología 8 (1): 7-20.

Nina D. A., G. Quispe, M. Romero, y P. Fierro. 2016 "Avances y progresos de las políticas y estrategias de seguridad alimentaria en Ecuador". Revista Investigaciones Altoandinas, 18 (2): 213-222

Bedogni M. C., S Capezio, y M. Huarte. 2009. "Comportamiento frente a estrés hídrico de variedades nativas y especies silvestres de papa". Revista Latinoamericana de la Papa 15 (1): $72-75$.

Gabriel. 2013."Respuesta de variedades mejoradas de papa (Solanum tuberosum L.) al estrés hídrico por sequía." Journal of the Selva Andina Biosphere $1(1): 33-44$. 
Gabriel J., S. Veramendi, A. Angulo, y J. Magne. 2013.

"Respuesta de variedades de papa (Solanum tuberosum L.) al estrés hídrico por sequía". Journal of the selva andina Biosphere 1 (1): 25-36.

Haapala T. 2008. Producción de tubérculos semilla libres de enfermedades. http://www.fao.org/potato-2008/pdf/IYP9es.pdf (Consultado el 14 de octubre del 2017)

Herrera M. C., A. Rueda, y C. Pinzón. 2012 "Percepciones sobre los fenómenos de variabilidad climática y cambio climático entre campesinos del centro de Santander, Colombia." Ambiente y Desarrollo 16(31): 25-37.

Hinojosa, S., L. A. Rivadeneira, J. Andrade y, H. Cuesta. 2010. "Estudio del Comportamiento Agronómico De Genotipos De Papa (Solanum SP.) Bajo Estrés Hídrico En Invernadero. ” I Congreso Internacional de Investigación y Desarrollo de Papas Nativas. Quito (Ecuador).

MAE (Ministerio del Ambiente). 2012. "Sistema de clasificación de los ecosistemas del Ecuador continental." Ecuador (Quito).

Morton, J. F. 2007. "The impact of climate change on smallholder and subsistence agriculture." Proceedings of the National Academy of Sciences 104 (50): 19680-19685. DOI: 10.1073/pnas.0701855104

Polanía, J., M. Rao Idupulapati, B. Steve, R. García. 2009 "Desarrollo y distribución de raíces bajo estrés por sequía en fríjol común (Phaseolus vulgaris L.) En un sistema de tubos con suelo." Agronomía Colombiana 27 (1): 25-32.

Quintana-Rodríguez, L 2018. Efecto de la disponibilidad hídrica y aumento de temperatura en la calidad nutricional de tubérculos de papa (Solanum tuberosum L.). Tesis de Grado. Universidad Austral de Chile. Valdivia (Chile). 